\title{
Effects of phonon-phonon coupling on low-lying states in neutron-rich Sn isotopes.
}

\author{
A.P. Severyukhin \\ Bogoliubov Laboratory of Theoretical Physics, Joint Institute for Nuclear Research, 141980 \\ Dubna, Moscow region, Russia \\ V.V. Voronov \\ Bogoliubov Laboratory of Theoretical Physics, Joint Institute for Nuclear Research, 141980 \\ Dubna, Moscow region, Russia \\ Nguyen Van Giai \\ Institut de Physique Nucléaire, CNRS-IN2P3, Université Paris-Sud, F-91406 Orsay Cedex, \\ France
}

\begin{abstract}
Starting from an effective Skyrme interaction we present a method to take into account the coupling between one- and two-phonon terms in the wave functions of excited states. The approach is a development of a finite rank separable approximation for the quasiparticle RPA calculations proposed in our previous work. The influence of the phonon-phonon coupling on energies and transition probabilities for the low-lying quadrupole and octupole states in the neutron-rich Sn isotopes is studied.
\end{abstract}

Typeset using REVTEX 


\section{INTRODUCTION}

The experimental and theoretical studies of properties of the excited states in nuclei far from the $\beta$-stability line are presently the object of very intensive activity. The random phase approximation (RPA) [1-4] is a well-known and successful way to treat nuclear vibrational excitations. Using the Gogny's [5] or Skyrme-type [6] effective nucleon-nucleon interactions the most consistent models can describe the ground states in the framework of the Hartree-Fock (HF) and Hartree-Fock-Bogoliubov (HFB) approximations and the excited states within the RPA and quasiparticle RPA (QRPA). Such models are quite successful not only to reproduce the nuclear ground state properties $[7,8]$, but also to describe the main features of nuclear excitations in closed-shell $[9,10]$ and open-shell nuclei [11-14]. In the latter case the pairing correlations are very important.

Due to the anharmonicity of vibrations there is a coupling between one-phonon and more complex states $[2,4]$ and the complexity of calculations beyond standard RPA or QRPA increases rapidly with the size of the configuration space, so one has to work within limited spaces. Making use of separable forces one can perform calculations of nuclear characteristics in very large configuration spaces since there is no need to diagonalize matrices whose dimensions grow with the size of configuration space. For example, the well-known quasiparticle-phonon model (QPM) [4] can do very detailed predictions for nuclei away from

closed shells [15], but it is very difficult to extrapolate the phenomenalogical parameters of the nuclear hamiltonian to new regions of nuclei.

That is why a finite rank approximation for the particle-hole $(\mathrm{p}-\mathrm{h})$ interaction resulting from the Skyrme forces has been suggested in our previous work [16]. Thus, the selfconsistent mean field can be calculated with the original Skyrme interaction whereas the RPA solutions would be obtained with the finite rank approximation to the $\mathrm{p}$-h matrix elements. It was found that the finite rank approximation can reproduce reasonably well the dipole and quadrupole strength distributions in Ar isotopes. Alternative schemes to factorize the $\mathrm{p}-\mathrm{h}$ interaction were considered in [17-19]. 
Recently, the finite rank approximation for $\mathrm{p}$-h interactions of Skyrme type has been generalized to take into account the pairing correlations [20]. The QRPA was used to describe characteristics of the low-lying $2^{+}$and $3^{-}$states and giant resonances in nuclei with very different mass numbers [20,21]. It was found that there is room for the phonon-phonon coupling effects in many cases. The first calculation to estimate this effect has been done for ${ }^{112} \mathrm{Sn}$ in $[22]$.

In the present work, we extend our approach to take into account the coupling between the one- and two-phonon terms in the wave functions of excited states. As an application of the method we present results for low-lying $2^{+}$and $3^{-}$states in neutron-rich Sn isotopes and compare them with recent experimental data [23] and other calculations [24-26].

This paper is organized as follows: in Sec. II we sketch our method allowing to consider effects of the phonon-phonon coupling. In Sec. III we discuss details of calculations and show how this approach can be applied to treat the low-lying collective states. Results of calculations for properties of the quadrupole and octupole states in ${ }^{124-134} \mathrm{Sn}$ isotopes are given in Sec.IV. Conclusions are drawn in Section V.

\section{METHOD OF CALCULATIONS}

\section{A. The model hamiltonian and QRPA}

We start from the effective Skyrme interaction [6] and use the notation of Ref. [27] containing explicit density dependence and all spin-exchange terms. The single-particle spectrum is calculated within the HF method. The continuous part of the single-particle spectrum is discretized by diagonalizing the HF hamiltonian on a harmonic oscillator basis [28]. The p-h residual interaction $\tilde{V}_{\text {res }}$ corresponding to the Skyrme force and including both direct and exchange terms can be obtained as the second derivative of the energy density functional with respect to the density [29]. Following our previous papers [16] we simplify $\tilde{V}_{\text {res }}$ by approximating it by its Landau-Migdal form. For Skyrme interactions all Landau 
parameters $F_{l}, G_{l}, F_{l}^{\prime}, G_{l}^{\prime}$ with $l>1$ are zero. Here, we keep only the $l=0$ terms in $V_{\text {res }}$ and in the coordinate representation one can write it in the following form:

$$
V_{\text {res }}\left(\mathbf{r}_{1}, \mathbf{r}_{2}\right)=N_{0}^{-1}\left[F_{0}\left(r_{1}\right)+G_{0}\left(r_{1}\right) \sigma_{1} \cdot \sigma_{2}+\left(F_{0}^{\prime}\left(r_{1}\right)+G_{0}^{\prime}\left(r_{1}\right) \sigma_{1} \cdot \sigma_{2}\right) \tau_{1} \cdot \tau_{2}\right] \delta\left(\mathbf{r}_{1}-\mathbf{r}_{2}\right)
$$

where $\sigma_{i}$ and $\tau_{i}$ are the spin and isospin operators, and $N_{0}=2 k_{F} m^{*} / \pi^{2} \hbar^{2}$ with $k_{F}$ and $m^{*}$ standing for the Fermi momentum and nucleon effective mass. The expressions for $F_{0}, G_{0}, F_{0}^{\prime}, G_{0}^{\prime}$ in terms of the Skyrme force parameters can be found in Ref. [27]. Because of the density dependence of the interaction the Landau parameters of Eq.(1) are functions of the coordinate $\mathbf{r}$.

In what follows we use the second quantized representation and $V_{\text {res }}$ can be written as:

$$
\hat{V}_{\text {res }}=\frac{1}{2} \sum_{1234} V_{1234}: a_{1}^{+} a_{2}^{+} a_{4} a_{3}:
$$

where $a_{1}^{+}\left(a_{1}\right)$ is the particle creation (annihilation) operator and 1 denotes the quantum numbers $\left(n_{1} l_{1} j_{1} m_{1}\right)$,

$$
V_{1234}=\int \phi_{1}^{*}\left(\mathbf{r}_{1}\right) \phi_{2}^{*}\left(\mathbf{r}_{2}\right) V_{r e s}\left(\mathbf{r}_{1}, \mathbf{r}_{2}\right) \phi_{3}\left(\mathbf{r}_{1}\right) \phi_{4}\left(\mathbf{r}_{2}\right) \mathbf{d} \mathbf{r}_{1} \mathbf{d} \mathbf{r}_{2}
$$

After integrating over the angular variables one needs to calculate the radial integrals. As it is shown in $[16,20]$ the radial integrals can be calculated accurately by choosing a large enough cutoff radius $R$ and using a $N$-point integration Gauss formula with abscissas $r_{k}$ and weights $w_{k}$. Thus, the two-body matrix element is a sum of $N$ separable terms, i.e., the residual interaction takes the form of a rank $N$ separable interaction.

We employ a hamiltonian including an average HF field, pairing interactions, the isoscalar and isovector particle-hole $(\mathrm{p}-\mathrm{h})$ residual forces in a finite rank separable form [20]:

$$
H=\sum_{\tau}\left(\sum_{j m}^{\tau}\left(E_{j}-\lambda_{\tau}\right) a_{j m}^{\dagger} a_{j m}-\frac{1}{4} V_{\tau}^{(0)}: P_{0}^{\dagger}(\tau) P_{0}(\tau):\right)+\hat{V}_{r e s}
$$

where

$$
P_{0}^{+}(\tau)=\sum_{j m}^{\tau}(-1)^{j-m} a_{j m}^{+} a_{j-m}^{+}
$$


We sum over the $\operatorname{proton}(p)$ and neutron $(n)$ indexes and the notation $\{\tau=(n, p)\}$ is used. A change $\tau \leftrightarrow-\tau$ means a change $p \leftrightarrow n$. The single-particle states are specified by the quantum numbers $(j m), E_{j}$ are the single-particle energies, $\lambda_{\tau}$ the chemical potentials. $V_{\tau}^{(0)}$ is the interaction strength in the particle-particle channel. The hamiltonian (4) has the same form as the QPM hamiltonian with $N$ separable terms $[4,30]$, but the single-particle spectrum and parameters of the $\mathrm{p}$-h residual interaction are calculated making use of the Skyrme forces.

In what follows we work in the quasiparticle representation defined by the canonical Bogoliubov transformation:

$$
a_{j m}^{+}=u_{j} \alpha_{j m}^{+}+(-1)^{j-m} v_{j} \alpha_{j-m} .
$$

The hamiltonian (4) can be represented in terms of bifermion quasiparticle operators and their conjugates [4]:

$$
\begin{gathered}
B\left(j j^{\prime} ; \lambda \mu\right)=\sum_{m m^{\prime}}(-1)^{j^{\prime}+m^{\prime}}\left\langle j m j^{\prime} m^{\prime} \mid \lambda \mu\right\rangle \alpha_{j m}^{+} \alpha_{j^{\prime}-m^{\prime}} \\
A^{+}\left(j j^{\prime} ; \lambda \mu\right)=\sum_{m m^{\prime}}\left\langle j m j^{\prime} m^{\prime} \mid \lambda \mu\right\rangle \alpha_{j m}^{+} \alpha_{j^{\prime} m^{\prime}}^{+} .
\end{gathered}
$$

We introduce the phonon creation operators

$$
Q_{\lambda \mu i}^{+}=\frac{1}{2} \sum_{j j^{\prime}}\left(X_{j j^{\prime}}^{\lambda i} A^{+}\left(j j^{\prime} ; \lambda \mu\right)-(-1)^{\lambda-\mu} Y_{j j^{\prime}}^{\lambda i} A\left(j j^{\prime} ; \lambda-\mu\right)\right) .
$$

where the index $\lambda$ denotes total angular momentum and $\mu$ is its z-projection in the laboratory system. One assumes that the ground state is the QRPA phonon vacuum $|0\rangle$, i.e. $Q_{\lambda \mu i}|0\rangle=0$. We define the excited states for this approximation by $Q_{\lambda \mu i}^{+}|0\rangle$. The quasiparticle energies $\left(\varepsilon_{j}\right)$, the chemical potentials $\left(\lambda_{\tau}\right)$, the energy gap and the coefficients $u, v$ of the Bogoliubov transformations (6) are determined from the BCS equations with the single-particle spectrum that is calculated within the HF method with the effective Skyrme interaction. Making use of the linearized equation-of-motion approach [1]:

$$
\left\langle 0\left|\left[\delta Q_{\lambda \mu i},\left[H, Q_{\lambda \mu i}^{+}\right]\right]\right| 0\right\rangle=\omega_{\lambda i}\left\langle 0\left|\left[\delta Q_{\lambda \mu i}, Q_{\lambda \mu i}^{+}\right]\right| 0\right\rangle
$$


with the normalization condition:

$$
\left\langle 0\left|\left[Q_{\lambda \mu i}, Q_{\lambda \mu i^{\prime}}^{+}\right]\right| 0\right\rangle=\delta_{i i^{\prime}}
$$

one can get the QRPA equations $[3,4]$ :

$$
\left(\begin{array}{cc}
\mathcal{A} & \mathcal{B} \\
-\mathcal{B} & -\mathcal{A}
\end{array}\right)\left(\begin{array}{l}
X \\
Y
\end{array}\right)=w\left(\begin{array}{l}
X \\
Y
\end{array}\right) .
$$

In QRPA problems there appear two types of interaction matrix elements, the $A_{\left(j_{1} j_{1}^{\prime}\right)\left(j_{2} j_{2}^{\prime}\right)}^{(\lambda)}$ matrix related to forward-going graphs and the $B_{\left(j_{1} j_{1}^{\prime}\right)_{\tau}\left(j_{2} j_{2}^{\prime}\right)_{q}}^{(\lambda)}$ matrix related to backwardgoing graphs. Solutions of this set of linear equations yield the eigen-energies and the amplitudes $X, Y$ of the excited states. The dimension of the matrices $\mathcal{A}, \mathcal{B}$ is the space size of the two-quasiparticle configurations. For our case expressions for $\mathcal{A}, \mathcal{B}$ and $X, Y$ are given in [20]. Using the finite rank approximation we need to invert a matrix of dimension $4 N \times 4 N$ independently of the configuration space size [16,20]. Therefore, this approach enables one to reduce remarkably the dimensions of the matrices that must be inverted to perform structure calculations in very large configuration spaces.

\section{B. Phonon-phonon coupling}

Our calculations [20] show that, for the normal parity states one can neglect the spinmultipole terms of the $\mathrm{p}$-h residual interaction (1). Using the completeness and orthogonality conditions for the phonon operators one can express bifermion operators $A^{+}\left(j j^{\prime} ; \lambda \mu\right)$ and $A\left(j j^{\prime} ; \lambda \mu\right)$ through the phonon ones and the initial hamiltonian (4) can be rewritten in terms of quasiparticle and phonon operators in the following form:

$$
\begin{gathered}
H=h_{0}+h_{Q Q}+h_{Q B} \\
h_{0}=\sum_{j m} \varepsilon_{j} \alpha_{j m}^{+} \alpha_{j m}
\end{gathered}
$$




$$
\begin{gathered}
h_{Q Q}=-\frac{1}{4} \sum_{\lambda \mu i i^{\prime} \tau} W^{\lambda i i^{\prime}}(\tau) Q_{\lambda \mu i}^{+} Q_{\lambda \mu i^{\prime}} \\
h_{Q B}=-\frac{1}{2} \sum_{\lambda \mu i \tau} \sum_{j j^{\prime}}^{\tau} \Gamma_{j j^{\prime}}^{\lambda i}(\tau)\left((-)^{\lambda-\mu} Q_{\lambda \mu i}^{+}+Q_{\lambda-\mu i}\right) B\left(j j^{\prime} ; \lambda-\mu\right)+\text { h.c. }
\end{gathered}
$$

The coefficients $W, \Gamma$ of the hamiltonian (13) are sums of $\mathrm{N}$ combinations of phonon amplitudes, the Landau parameters, the reduced matrix element of the spherical harmonics and radial parts of the HF single-particle wave function (see Appendix A). It is worth to point out that the term $h_{Q B}$ is responsible for the mixing of the configurations and, therefore, for the description of many characteristics of the excited states of even-even nuclei [4].

To take into account the mixing of the configurations in the simplest case one can write the wave functions of excited states as:

$$
\Psi_{\nu}(\lambda \mu)=\left\{\sum_{i} R_{i}(\lambda \nu) Q_{\lambda \mu i}^{+}+\sum_{\lambda_{1} i_{1} \lambda_{2} i_{2}} P_{\lambda_{2} i_{2}}^{\lambda_{1} i_{1}}(\lambda \nu)\left[Q_{\lambda_{1} \mu_{1} i_{1}}^{+} Q_{\lambda_{2} \mu_{2} i_{2}}^{+}\right]_{\lambda \mu}\right\}|0\rangle
$$

with the normalization condition:

$$
\sum_{i} R_{i}^{2}(J \nu)+2 \sum_{\lambda_{1} i_{1} \lambda_{2} i_{2}}\left(P_{\lambda_{2} i_{2}}^{\lambda_{1} i_{1}}(J \nu)\right)^{2}=1
$$

Using the variational principle in the form:

$$
\delta\left(\left\langle\Psi_{\nu}(\lambda \mu)|H| \Psi_{\nu}(\lambda \mu)\right\rangle-E_{\nu}\left(\left\langle\Psi_{\nu}(\lambda \mu) \mid \Psi_{\nu}(\lambda \mu)\right\rangle-1\right)\right)=0,
$$

one obtains a set of linear equations for the unknown amplitudes $R_{i}(J \nu)$ and $P_{\lambda_{2} i_{2}}^{\lambda_{1} i_{1}}(J \nu)$ :

$$
\begin{gathered}
\left(\omega_{J i}-E_{\nu}\right) R_{i}(J \nu)+\sum_{\lambda_{1} i_{1} \lambda_{2} i_{2}} U_{\lambda_{2} i_{2}}^{\lambda_{1} i_{1}}(J \nu) P_{\lambda_{2} i_{2}}^{\lambda_{1} i_{1}}(J \nu)=0 \\
\sum_{i} U_{\lambda_{2} i_{2}}^{\lambda_{1} i_{1}}(J i) R_{i}(J \nu)+2\left(\omega_{\lambda_{1} i_{1}}+\omega_{\lambda_{2} i_{2}}-E_{\nu}\right) P_{\lambda_{2} i_{2}}^{\lambda_{1} i_{1}}(J \nu)=0
\end{gathered}
$$

$U_{\lambda_{2} i_{2}}^{\lambda_{1} i_{1}}(J i)$ is the matrix element coupling one- and two-phonon configurations [4,31]:

$$
U_{\lambda_{2} i_{2}}^{\lambda_{1} i_{1}}(J i)=\left\langle 0\left|Q_{J i} h_{Q B}\left[Q_{\lambda_{1} i_{1}}^{+} Q_{\lambda_{2} i_{2}}^{+}\right]_{J}\right| 0\right\rangle
$$


The expression of $U_{\lambda_{2} i_{2}}^{\lambda_{1} i_{1}}(J i)$ is given in Appendix B. The number of linear equations (20), (21) equals the number of one- and two-phonon configurations included in the wave function (17).

The energies of excited states $E_{\nu}$ are solutions of the secular equation

$$
F\left(E_{\nu}\right) \equiv \operatorname{det}\left|\left(\omega_{\lambda i}-E_{\nu}\right) \delta_{i i^{\prime}}-\frac{1}{2} \sum_{\lambda_{1} i_{1}, \lambda_{2} i_{2}} \frac{U_{\lambda_{2} i_{2}}^{\lambda_{1} i_{1}}(\lambda i) U_{\lambda_{2} i_{2}}^{\lambda_{1} i_{1}}\left(\lambda i^{\prime}\right)}{\omega_{\lambda_{1} i_{1}}+\omega_{\lambda_{2} i_{2}}-E_{\nu}}\right|=0,
$$

where the rank of the determinant equals the number of the one-phonon configurations. Using Eqs.(20), (21) and the normalization condition (18), one can find the amplitudes $R_{i}(J \nu)$ and $P_{\lambda_{2} i_{2}}^{\lambda_{1} i_{1}}(J \nu)$.

It is necessary to point out that the equations derived above have the same form as the basic QPM equations [4,31], but the single-particle spectrum and the p-h residual interaction are determined making use of the Skyrme interactions.

\section{DETAILS OF CALCULATIONS}

We apply the present approach to study characteristics of the low-lying vibrational states in the neutron-rich Sn isotopes. In this paper we use the parametrization SLy4 [32] of the Skyrme interaction. This parametrization was proposed to describe isotopic properties of nuclei from the $\beta$-stability line to the drip lines. Spherical symmetry is assumed for the HF ground states.

The pairing constants $V_{\tau}^{0}$ are fixed to reproduce the odd-even mass difference of neighboring nuclei. It is well known $[11,12]$ that the constant gap approximation leads to an overestimating of occupation probabilities for subshells that are far from the Fermi level and it is necessary to introduce a cut-off in the single-particle space. Above this cut-off subshells don't participate in the pairing effect. In our calculations we choose the BCS subspace to include all subshells lying below $5 \mathrm{MeV}$.

In order to perform QRPA calculations, the single-particle continuum is discretized [28] by diagonalizing the HF hamiltonian on a basis of twelve harmonic oscillator shells and 
cutting off the single-particle spectra at the energy of $100 \mathrm{MeV}$. This is sufficient to exhaust practically all the energy-weighted sum rule.

The Landau parameters $F_{0}, G_{0}, F_{0}^{\prime}, G_{0}^{\prime}$ expressed in terms of the Skyrme force parameters [27] depend on $k_{F}$. As it is pointed out in our previous works [16,20] one needs to adopt some effective value for $k_{F}$ to give an accurate representation of the original $\mathrm{p}$-h Skyrme interaction. For the present calculations we use the nuclear matter value for $k_{F}$.

Our previous investigations [20] enable us to conclude that $N=45$ for the rank of our separable approximation is enough for multipolarities $\lambda \leq 3$ in nuclei with $A \leq 208$. Increasing $N$, for example, up to $N=60$ in ${ }^{208} \mathrm{~Pb}$ changes results for energies and transition probabilities not more than by $1 \%$. Our calculations show that, for the natural parity states one can neglect the spin-multipole interactions and this reduces by a factor 2 the total matrix dimension, i.e., the matrix dimensions never exceed $2 N \times 2 N$ independently of the configuration space size $[16,20]$.

The two-phonon configurations of the wave function (17) are constructed from natural parity phonons with multipolarities $\lambda=2,3,4,5$. All one-phonon configurations with energies below $8 \mathrm{MeV}$ for ${ }^{124-130,134} \mathrm{Sn}$ and $10 \mathrm{MeV}$ for ${ }^{132} \mathrm{Sn}$ are included in the the wave function (17). The cut-off in the space of the two-phonon configurations is $21 \mathrm{MeV}$. An extension of the space for one- and two-phonon configurations does not change results for energies and transition probabilities practically.

\section{RESULTS OF CALCULATIONS}

As an application of the method we investigate effects of the phonon-phonon coupling on energies and transition probabilities to $2_{1}^{+}$and $3_{1}^{-}$states in ${ }^{124-134} \mathrm{Sn}$.

Results of our calculations for the $2_{1}^{+}$energies and transition probabilities $B(E 2)$ are compared with experimental data $[23,33]$ in Table I. Columns "QRPA" and "2PH" give values calculated within the QRPA and taking into account the phonon-phonon coupling, respectively. 
As it is seen from Table I there is a remarkable increase of the $2_{1}^{+}$energy and $B(E 2 \uparrow)$ in ${ }^{132} \mathrm{Sn}$ in comparison with those in ${ }^{130,134} \mathrm{Sn}$. Such a behaviour of $B(E 2 \uparrow)$ is related with the proportion between the QRPA amplitudes for neutrons and protons in Sn isotopes. The neutron amplitudes are dominant in all $\mathrm{Sn}$ isotopes and the contribution of the main neutron configuration $\left\{1 h_{11 / 2}, 1 h_{11 / 2}\right\}$ increases from $81.2 \%$ in ${ }^{124} \mathrm{Sn}$ to $92.8 \%$ in ${ }^{130} \mathrm{Sn}$ when neutrons fill the subshell $1 h_{11 / 2}$. At the same time the contribution of the main proton configuration $\left\{2 d_{5 / 2}, 1 g_{9 / 2}\right\}$ is decreasing from $9.3 \%$ in ${ }^{124} \mathrm{Sn}$ to $3.9 \%$ in ${ }^{130} \mathrm{Sn}$. The closure of the neutron subshell $1 h_{11 / 2}$ in ${ }^{132} \mathrm{Sn}$ leads to the vanishing of the neutron paring. The energy of the first neutron two-quasiparticle pole $\left\{2 f_{7 / 2}, 1 h_{11 / 2}\right\}$ in ${ }^{132} \mathrm{Sn}$ is greater than energies of the first poles in ${ }^{130,134} \mathrm{Sn}$ and the contribution of the $\left\{2 f_{7 / 2}, 1 h_{11 / 2}\right\}$ configuration in the doubly magic ${ }^{132} \mathrm{Sn}$ is about $61 \%$. Furthermore, the first pole in ${ }^{132} \mathrm{Sn}$ is closer to the proton poles. This means that the contribution of the proton two-quasiparticle configurations is greater than those in the neighbouring isotopes and as a result the main proton configuration $\left\{2 d_{5 / 2}, 1 g_{9 / 2}\right\}$ in ${ }^{132} \mathrm{Sn}$ exhausts about $33 \%$. In ${ }^{134} \mathrm{Sn}$ the leading contribution (about $99 \%$ ) comes from the neutron configuration $\left\{2 f_{7 / 2}, 2 f_{7 / 2}\right\}$ and as a result the $B(E 2)$ value is reduced. Such a behaviour of the $2_{1}^{+}$energies and $B(E 2)$ values in the neutron-rich $\mathrm{Sn}$ isotopes reflects the shell structure in this region. It is worth to mention that the first prediction of the anomalous behaviour of $2^{+}$excitations around ${ }^{132} \mathrm{Sn}$ based on the QRPA calculations with a separable quadrupole-plus-pairing hamiltonian has been done in [24]. Other QRPA calculations with Skyrme [13] and Gogny [26] forces give similar results for Sn isotopes.

One can see from Table I that the inclusion of the two-phonon terms results in a decrease of the energies and a reduction of transition probabilities. Note that the effect of the twophonon configurations is important for the energies and this effect becomes weak in ${ }^{132} \mathrm{Sn}$. There is some overestimate of the energies for the QRPA calculations and taking into account of the two-phonon terms improves the description of the $2_{1}^{+}$energies . The reduction of the $B(E 2)$ values is small in most cases due to the crucial contribution of the one-phonon configuration in the wave function structure. 
Results of our calculations for the $3_{1}^{-}$energies and the transition probabilities $B(E 3)$ compared to experimental data [34] are shown in Table II. As for the quadrupole excitations the influence of coupling between one- and two-phonon terms in the wave functions of the $3_{1}^{-}$states leads to the decrease of the energies and the reduction of transition probabilities. In spite of the fact that the $3_{1}^{-}$states have strong collectivity and many two-quasiparticle configurations give a contribution in the QRPA wave functions in Sn isotopes the phononphonon coupling is not very strong in this case. Our calculation shows that the main reason is the smallness of the matrix elements coupling the one-phonon configuration $\left\{3_{1}^{-}\right\}$and the two-phonon configuration $\left\{2_{1}^{+} ; 3_{1}^{-}\right\}\left(U_{3_{1}^{-}}^{2^{+}}\left(3_{1}^{-}\right)\right)$. As a result the decrease of the $3_{1}^{-}$energies is about $10 \%$. In the present paper we neglect the p-p channel that can be important for collective phonons and can reduce the collectivity of states [4,35]. This can give an additional lowering of energies and transition probabilities, but this is not the case for ${ }^{132} \mathrm{Sn}$. It is worth to mention that experimental data for $3_{1}^{-}$states in the neutron-rich Sn isotopes are very scarce.

An additional information about the structure of the first $2^{+}, 3^{-}$states can be extracted by looking at the ratio of the multipole transition matrix elements $M_{n} / M_{p}$ that depends on the relative contributions of the proton and neutron configurations. In the framework of the collective model for isoscalar excitations this ratio is equal to $M_{n} / M_{p}=N / Z$ and any deviation from this value can indicate an isovector character of the state. The $M_{n} / M_{p}$ ratio can be determined experimentally by using different external probes [36-38]. Our calculated values for the $M_{n} / M_{p}$ ratios for the $2_{1}^{+}$and $3_{1}^{-}$states are shown in Table III. The calculated $M_{n} / M_{p}$ ratios are rather close to $N / Z$ except $2_{1}^{+}$in ${ }^{134} \mathrm{Sn}$. It is worth to note that the deviation of the ratio for $2_{1}^{+}$state in ${ }^{132} \mathrm{Sn}$ correlates with the increase of the contribution of the proton two-quasiparticle configurations. 


\section{CONCLUSIONS}

A finite rank separable approximation for the QRPA calculations with Skyrme interactions that was proposed in our previous work is extended to take into account the coupling between one- and two-phonon terms in the wave functions of excited states. The suggested approach enables one to reduce remarkably the dimensions of the matrices that must be diagonalized to perform structure calculations in very large configuration spaces. As an application of the method we have studied the behavior of the energies and transition probabilities to $2_{1}^{+}$and $3_{1}^{-}$states in ${ }^{124-134} \mathrm{Sn}$. The inclusion of the two-phonon configurations results in a decrease of the energies and a reduction of transition probabilities. It is shown that there is some overestimate of $2_{1}^{+}$energies for the QRPA calculations and the effect of the two-phonon configurations is important, but this effect decreases in ${ }^{132} \mathrm{Sn}$. The inclusion of the two-phonon terms does not change the effect of a remarkable increase of the

QRPA value of $B\left(E 2 ; 0^{+} \rightarrow 2_{1}^{+}\right)$for the doubly-closed shell nucleus ${ }^{132} \mathrm{Sn}$ in comparison with its neighbors. A systematical study of the influence of the two-phonon terms taking into account the p-p channel on properties of the low-lying states is now in progress.

\section{ACKNOWLEDGMENTS}

We are grateful to Prof. Ch.Stoyanov for valuable discussions and help. A.P.S. and V.V.V. thank the hospitality of IPN-Orsay where a part of this work was done. This work is partly supported by INTAS Fellowship grant for Young Scientists (Fellowship Reference N YSF 2002-70), and by IN2P3-JINR agreement.

\section{APPENDIX A}

The coefficients of the hamiltonian (13) are given by the following expressions:

$$
W^{\lambda i i^{\prime}}(\tau)=\sum_{k=1}^{N}\left(\frac{D_{M}^{\lambda i k}(\tau)}{\sqrt{2 \mathcal{Y}_{\tau}^{\lambda k i^{\prime}}}}+\frac{D_{M}^{\lambda i^{\prime} k}(\tau)}{\sqrt{2 \mathcal{Y}_{\tau}^{\lambda k i}}}\right)
$$




$$
\Gamma_{j j^{\prime}}^{\lambda i}(\tau)=\sum_{k=1}^{N} \frac{f_{j j^{\prime}}^{(\lambda k)} v_{j j^{\prime}}^{(-)}}{\sqrt{2 \mathcal{Y}_{\tau}^{\lambda k i}}}
$$

where

$$
\begin{gathered}
D_{M}^{\lambda i k}(\tau)=\sum_{j j^{\prime}}^{\tau} f_{j j^{\prime}}^{(\lambda k)} u_{j j^{\prime}}^{(+)}\left(X_{j j^{\prime}}^{\lambda i}+Y_{j j^{\prime}}^{\lambda i}\right), \\
\mathcal{Y}_{\tau}^{\lambda k i}=\frac{2(2 \lambda+1)^{2}}{\left(D_{M}^{\lambda i k}(\tau)\left(\kappa_{0}^{(M, k)}+\kappa_{1}^{(M, k)}\right)+D_{M}^{\lambda i k}(-\tau)\left(\kappa_{0}^{(M, k)}-\kappa_{1}^{(M, k)}\right)\right)^{2}}, \\
v_{j j^{\prime}}^{(-)}=u_{j} u_{j^{\prime}}-v_{j} v_{j^{\prime}} u_{j j^{\prime}}^{(+)}=u_{j} v_{j^{\prime}}+v_{j} u_{j^{\prime}} .
\end{gathered}
$$

In the above expressions, $f_{j j^{\prime}}^{(\lambda k)}$ denotes the single-particle radial matrix elements [20]:

$$
f_{j_{1} j_{2}}^{(\lambda k)}=u_{j_{1}}\left(r_{k}\right) u_{j_{2}}\left(r_{k}\right) i^{\lambda}\left\langle j_{1}\left\|Y_{\lambda}\right\| j_{2}\right\rangle
$$

where $u_{j_{1}}\left(r_{k}\right)$ is the radial part of the HF single-particle wavefunction at the abscissas of the $N$-point integration Gauss formula $r_{k} \cdot \kappa_{0}^{(M, k)}$ and $\kappa_{1}^{(M, k)}$ are defined by the Landau parameters as

$$
\left(\begin{array}{c}
\kappa_{0}^{(M, k)} \\
\kappa_{1}^{(M, k)}
\end{array}\right)=-N_{0}^{-1} \frac{R w_{k}}{2 r_{k}^{2}}\left(\begin{array}{c}
F_{0}\left(r_{k}\right) \\
F_{0}^{\prime}\left(r_{k}\right)
\end{array}\right)
$$

\section{APPENDIX B}

The matrix elements $U_{\lambda_{2} i_{2}}^{\lambda_{1} i_{1}}(J i)$ have the following form:

$$
\begin{aligned}
U_{\lambda_{2} i_{2}}^{\lambda_{1} i_{1}}(\lambda i)=( & -1)^{\lambda_{1}+\lambda_{2}+\lambda} \sqrt{\left(2 \lambda_{1}+1\right)\left(2 \lambda_{2}+1\right)} \sum_{\tau} \sum_{j_{1} j_{2} j_{3}}{ }^{\tau} \times \\
& \left(\Gamma_{j_{1} j_{2}}^{\lambda i}(\tau)\left\{\begin{array}{ccc}
\lambda_{1} & \lambda_{2} & \lambda \\
j_{2} & j_{1} & j_{3}
\end{array}\right\}\left(X_{j_{2} j_{3}}^{\lambda_{2} i_{2}} Y_{j_{3} j_{1}}^{\lambda_{1} i_{1}}+X_{j_{3} j_{1}}^{\lambda_{1} i_{1}} Y_{j_{2} j_{3}}^{\lambda_{2} i_{2}}\right)+\right. \\
& \Gamma_{j_{1} j_{2}}^{\lambda_{1} i_{1}}(\tau)\left\{\begin{array}{ccc}
\lambda_{1} & \lambda_{2} & \lambda \\
j_{3} & j_{2} & j_{1}
\end{array}\right\}\left(Y_{j_{3} j_{1}}^{\lambda_{2} i_{2}} Y_{j_{2} j_{3}}^{\lambda i}+X_{j_{2} j_{3}}^{\lambda i} X_{j_{3} j_{1}}^{\lambda_{2} i_{2}}\right)+ \\
& \left.\Gamma_{j_{1} j_{2}}^{\lambda_{2} i_{2}}(\tau)\left\{\begin{array}{ccc}
\lambda_{1} & \lambda_{2} & \lambda \\
j_{1} & j_{3} & j_{2}
\end{array}\right\}\left(Y_{j_{2} j_{3}}^{\lambda_{1} i_{1}} Y_{j_{3} j_{1}}^{\lambda i}+X_{j_{3} j_{1}}^{\lambda i} X_{j_{2} j_{3}}^{\lambda_{1} i_{1}}\right)\right)
\end{aligned}
$$




\section{REFERENCES}

[1] D.J.Rowe, Nuclear Collective Motion, Models and Theory (Barnes and Noble, 1970).

[2] A. Bohr and B. Mottelson, Nuclear Structure vol.2 (Benjamin, New York, 1975).

[3] P.Ring and P.Schuck, The Nuclear Many Body Problem (Springer, Berlin, 1980).

[4] V.G. Soloviev, Theory of Atomic Nuclei: Quasiparticles and Phonons (Institute of Physics, Bristol and Philadelphia, 1992).

[5] D. Gogny, in Nuclear Self-consistent Fields, eds. G. Ripka and M. Porneuf (NorthHolland, Amsterdam, 1975).

[6] D. Vautherin and D.M. Brink, Phys. Rev. C 5, 626 (1972).

[7] H. Flocard and P. Quentin, Ann. Rev. Nucl. Part. Sci. 28, 523 (1978).

[8] J. Dobaczewski, W. Nazarewicz, T.R. Werner, J.F. Berger, C.R. Chinn and J. Dechargé, Phys. Rev. C 53, 2809 (1996).

[9] G. Colò, P.F. Bortignon, N. Van Giai, A. Bracco and R.A. Broglia, Phys. Lett. B276, 279 (1992).

[10] G. Colò, N. Van Giai, P.F. Bortignon and R.A. Broglia, Phys. Rev. C50, 1496 (1994).

[11] E. Khan and Nguyen Van Giai, Phys. Lett. B 472, 253 (2000).

[12] G. Colò, N. Van Giai, P.F. Bortignon and M.R. Quaglia, Phys. Lett. B485, 362 (2000).

[13] G. Colò and P.F. Bortignon, Nucl. Phys. A696, 427 (2001).

[14] E. Khan, N. Sandulescu, M. Grasso and Nguyen Van Giai, Phys. Rev. C66,024309 (2002).

[15] S. Galès, Ch. Stoyanov and A.I. Vdovin, Phys. Rep. 166, 127 (1988).

[16] Nguyen Van Giai, Ch. Stoyanov and V.V. Voronov, Phys. Rev. C57,1204 (1998). 
[17] T. Suzuki and H. Sagawa, Prog. Theor. Phys., 65, 565 (1981).

[18] P. Sarriguren, E. Moya de Guerra and A. Escuderos, Nucl. Phys., A 658, 13 (1999).

[19] V.O. Nesterenko, J. Kvasil and P.-G. Reinhard, Phys. Rev. C66, 044307 (2002).

[20] A.P. Severyukhin, Ch. Stoyanov, V.V. Voronov and Nguyen Van Giai, Phys. Rev. C66, $034304(2002)$.

[21] A.P. Severyukhin, V.V. Voronov, Ch. Stoyanov and Nguyen Van Giai, Physics of Atomic Nuclei 66, 1434 (2003).

[22] A.P. Severyukhin, V.V. Voronov, Ch. Stoyanov and Nguyen Van Giai, Nucl. Phys. A722, 123c, (2003).

[23] D.C. Radford et al., Phys. Rev. Lett. 88, 22501 (2002); Eur. Phys. J. A 15, 171 (2002); http:// www.phy.ornl.gov, HRIBF Newsletter, July 2003.

[24] J. Terasaki, J. Engel, W. Nazarewicz and M. Stoitsov, Phys. Rev. C66, 054313 (2002).

[25] G. Colò, P.F. Bortignon, D. Sarchi, D. T. Khoa, E. Khan and Nguyen Van Giai, Nucl. Phys. A722, 111c (2003).

[26] G. Giambrone et al., Nucl. Phys. A726, 3 (2003).

[27] Nguyen Van Giai and H. Sagawa, Phys. Lett. 106 B, 379 (1981).

[28] J.P. Blaizot and D. Gogny, Nucl. Phys. A284, 429 (1977).

[29] G.F. Bertsch and S.F. Tsai, Phys. Reports 18 C, 126 (1975).

[30] V.G. Soloviev, Yad. Fiz. 50, 40 (1989).

[31] V.V. Voronov and V.G. Soloviev, Phys. of Part. \& Nucl. 14583 (1983).

[32] E. Chabanat, P. Bonche, P. Haensel, J. Meyer and R. Schaeffer, Nucl. Phys. A 635, 231 (1998). 
[33] S. Raman, C.W. Nestor Jr. and P. Tikkanen, At. Data and Nucl. Data Tables 78, 1 (2001).

[34] T. Kibedi and R.H. Spear, At. Data and Nucl. Data Tables 80, 35 (2002).

[35] T. Kubo, H. Sakamoto, T. Kammuri and T. Kishimoto, Phys. Rev., C 54, 2331 (1996).

[36] A.M. Bernstein, V.R. Brown and V.A. Madsen, Comments Nucl. Part. Phys., 11, 203 (1983).

[37] M.A. Kennedy, P.D. Cottle and K.W. Kemper, Phys. Rev., C 46, 1811 (1992).

[38] J.K. Jewell et al., Phys. Lett. B 454, 181 (1999). 


\section{TABLES}

TABLE I. Energies and B(E2)-values for up-transitions to the first $2^{+}$states

\begin{tabular}{|c|c|c|c|c|c|c|}
\hline \multirow[t]{3}{*}{ Nucleus } & \multicolumn{3}{|c|}{$\begin{array}{l}\text { Energy } \\
(\mathrm{MeV})\end{array}$} & \multicolumn{3}{|c|}{$\begin{array}{c}\mathrm{B}(\mathrm{E} 2 \uparrow) \\
\left(\mathrm{e}^{2} \mathrm{~b}^{2}\right)\end{array}$} \\
\hline & \multirow[t]{2}{*}{ Exp. } & \multicolumn{2}{|c|}{ Theory } & \multirow[t]{2}{*}{ Exp. } & \multicolumn{2}{|c|}{ Theory } \\
\hline & & QRPA & $2 \mathrm{PH}$ & & QRPA & $2 \mathrm{PH}$ \\
\hline${ }^{124} \mathrm{Sn}$ & 1.13 & 1.92 & 1.03 & $0.1660 \pm 0.0040$ & 0.177 & 0.151 \\
\hline${ }^{126} \mathrm{Sn}$ & 1.14 & 1.96 & 1.30 & $0.10 \pm 0.03$ & 0.149 & 0.133 \\
\hline${ }^{128} \mathrm{Sn}$ & 1.17 & 2.08 & 1.48 & $0.073 \pm 0.006$ & 0.111 & 0.100 \\
\hline${ }^{130} \mathrm{Sn}$ & 1.22 & 2.37 & 1.73 & $0.023 \pm 0.005$ & 0.064 & 0.058 \\
\hline${ }^{132} \mathrm{Sn}$ & 4.04 & 4.47 & 4.03 & $0.14 \pm 0.06$ & 0.136 & 0.129 \\
\hline${ }^{134} \mathrm{Sn}$ & 0.73 & 1.65 & 1.34 & $0.029 \pm 0.006$ & 0.016 & 0.015 \\
\hline
\end{tabular}


TABLE II. Energies and B(E3)-values for up-transitions to the first $3^{-}$states

\begin{tabular}{|c|c|c|c|c|c|c|}
\hline \multirow[t]{3}{*}{ Nucleus } & \multicolumn{3}{|c|}{$\begin{array}{l}\text { Energy } \\
(\mathrm{MeV})\end{array}$} & \multicolumn{3}{|c|}{$\begin{array}{c}\mathrm{B}(\mathrm{E} 3 \uparrow) \\
\left(\mathrm{e}^{2} \mathrm{~b}^{3}\right)\end{array}$} \\
\hline & \multirow[t]{2}{*}{ Exp. } & \multicolumn{2}{|c|}{ Theory } & \multirow[t]{2}{*}{ Exp. } & \multicolumn{2}{|c|}{ Theory } \\
\hline & & QRPA & $2 \mathrm{PH}$ & & QRPA & $2 \mathrm{PH}$ \\
\hline${ }^{124} \mathrm{Sn}$ & 2.60 & 3.64 & 3.25 & $0.073 \pm 0.010$ & 0.208 & 0.196 \\
\hline${ }^{126} \mathrm{Sn}$ & 2.72 & 4.16 & 3.76 & & 0.191 & 0.176 \\
\hline${ }^{128} \mathrm{Sn}$ & & 4.66 & 4.22 & & 0.181 & 0.161 \\
\hline${ }^{130} \mathrm{Sn}$ & & 5.17 & 4.75 & & 0.183 & 0.159 \\
\hline${ }^{132} \mathrm{Sn}$ & 4.35 & 5.66 & 5.36 & & 0.202 & 0.191 \\
\hline${ }^{134} \mathrm{Sn}$ & & 5.01 & 4.51 & & 0.128 & 0.111 \\
\hline
\end{tabular}

TABLE III. $\left(M_{n} / M_{p}\right) /(N / Z)$ ratios for the first $2^{+}, 3^{-}$states

\begin{tabular}{lcccccc}
\hline \hline State & ${ }^{124} \mathrm{Sn}$ & ${ }^{126} \mathrm{Sn}$ & ${ }^{128} \mathrm{Sn}$ & ${ }^{130} \mathrm{Sn}$ & ${ }^{132} \mathrm{Sn}$ & ${ }^{134} \mathrm{Sn}$ \\
\hline $2_{1}^{+}$ & 0.99 & 0.99 & 0.98 & 0.97 & 0.81 & 1.44 \\
$3_{1}^{-}$ & 0.94 & 0.92 & 0.89 & 0.86 & 0.83 & 0.91 \\
\hline \hline
\end{tabular}

